\title{
Nuclear Architecture of Mouse Spermatocytes: Chromosome Topology, Heterochromatin, and Nucleolus
}

\author{
Soledad Berrios \\ Programa Genética Humana, ICBM, Facultad de Medicina, Universidad de Chile, Santiago, Chile
}

\section{Keywords}

Meiotic prophase · Mus musculus domesticus · Nuclear architecture $\cdot$ Spermatocytes

\begin{abstract}
The nuclear organization of spermatocytes in meiotic prophase I is primarily determined by the synaptic organization of the bivalents that are bound by their telomeres to the nuclear envelope and described as arc-shaped trajectories through the 3D nuclear space. However, over this basic meiotic organization, a spermatocyte nuclear architecture arises that is based on higher-ordered patterns of spatial associations among chromosomal domains from different bivalents that are conditioned by the individual characteristics of chromosomes and the opportunity for interactions between their domains. Consequently, the nuclear architecture is species-specific and prone to modification by chromosomal rearrangements. This model is valid for the localization of any chromosomal domain in the meiotic prophase nucleus. However, constitutive heterochromatin plays a leading role in shaping nuclear territories. Thus, the nuclear localization of nucleoli depends on the position of NORs in nucleolar bivalents, but the association among nucleolar chromosomes mainly depends on the presence of constitu-
\end{abstract}

\section{KARGER}

(C) 2017 S. Karger AG, Basel

E-Mail karger@karger.com

www.karger.com/cgr tive heterochromatin that does not affect the expression of the ribosomal genes. Constitutive heterochromatin and nucleoli form complex nuclear territories whose distribution in the nuclear space is nonrandom, supporting the hypothesis regarding the existence of a species-specific nuclear architecture in first meiotic prophase spermatocytes.

(c) 2017 S. Karger AG, Basel

\section{Meiotic Prophase I}

Meiosis generates gametes with half the chromosome complement of the original progenitor cell. This reduction in genetic content is accomplished by a single round of DNA replication followed by 2 successive rounds of chromosome segregation. Homologous chromosomes segregate to opposite poles at meiosis I, and then chromatids separate to opposite poles in meiosis II. During meiosis, 2 types of genetic recombination are present between paternal and maternal homologous chromosomes: intrachromosomal recombination that occurs through cross-overs at meiotic prophase I and the recombination that occurs as a consequence of the random distribution of homologous chromosomes at metaphase I. Both generate genetic variation in the resulting gametes, which

Soledad Berrios

Programa Genética Humana, ICBM Facultad de Medicina, Universidad de Chile 1027 Independencia Ave, Santiago 8380453 (Chile)

E-Mail sberrios@med.uchile.cl 
strongly supports the biological individuality of sexually reproduced organisms.

During meiotic prophase I, homologous chromosomes become paired and then synapse through a synaptonemal complex (SC). Initially, axial elements (AE) are formed along chromosomal cores between sister chromatids, and telomeres are attached to the nuclear envelope [Scherthan, 2001; Page and Hawley, 2004]. The following telomere-led nuclear movement and polarized chromosome arrangement (i.e., bouquet) facilitate chromosome alignment and homolog pairing/synapsis [Page and Hawley, 2004; Scherthan, 2009; Hiraoka and Dernburg, 2009; Shibuya and Watanabe, 2014; Zickler and Kleckner, 2016]. In most organisms, the protein Spo11 initiates meiotic recombination by generating DNA doublestrand breaks (DSBs), which are also crucial for successful synapses between homologous chromosomes [Baudat et al., 2013]. Strikingly, in the mouse, homolog recognition is mediated by the meiosis-specific cohesin rather than a DSB-dependent sequence search [Ishiguro et al., 2014]. Thus, the DSB-triggered homology search may mainly serve to proofread and stabilize pre-DSB pairing of homologous chromosomes [Boateng et al., 2013; Ishiguro et al., 2014]. Some reports suggest that the telomere is the initiation site for chromosome pairing/synapsis in mouse meiosis [Scherthan, 2001; Ding et al., 2007; Shibuya and Watanabe, 2014], whereas others suggest interstitial sites along the entire chromosome length [Bisig et al., 2012; Qiao et al., 2012; Boateng et al., 2013]. From the synapsis initiation sites, SC formation spreads in both directions along the entire length of the paired chromosomes [Bolcun-Filas et al., 2007; Hamer et al., 2008; Davies et al., 2012].

The SC is a proteinaceous structure that is assembled between the AE of the 2 homologues that consist of 2 lateral elements (LEs) that are bound in a zipper-like manner by the central element (CE) and the transverse filaments [Yang and Wang, 2009]. The chromatin, which is organized in loops, is connected to the LEs [Heyting, 1996; Page and Hawley, 2004; Fraune et al., 2012; Zickler and Kleckner, 2015]. Among several proteins that form part of the SC, the SYCP3 and SYCP2 proteins are the main constituents of the LEs [Lammers et al., 1994; Offenberg et al., 1998], whereas the SYCP1 protein is the main constituent of the transverse filaments [Meuwissen et al., 1992]. The C-terminal ends of SYCP1 interact with LEs, whereas the N-termini of opposing SYCP1 molecules bind head-to-head within the center of the SC, thereby mediating synapsis of the homologues [Fraune et al., 2012].
In pachytene spermatocytes autosomal homologous chromosomes are synapsed along their entire structure forming bivalents, and chromatin is decondensed and transcriptionally active [Geremia et al., 1977; Mahadevaiah et al., 2009]. The $\mathrm{X}$ and $\mathrm{Y}$ chromosomes only synapse and recombine through a short segment of sequence homology known as the pseudoautosomal region or PAR [Burgoyne, 1982; Hale et al., 1991]. Chromatin associated to unsynapsed chromosomal axes remains condensed [Solari, 1974] and transcriptionally inactive via a phenomenon that has been named meiotic sex chromosome inactivation [Turner, 2007; Burgoyne et al., 2009].

The tripartite structure of the SC, because of its $100 \mathrm{~nm}$ width, is only visible by electron microscopy. However, the longitudinal path of each SC into the nuclear space is only revealed by $3 \mathrm{D}$ reconstruction of serial sectioning of a pachytene nucleus [Wettstein and Sotelo, 1967; von Wettstein et al., 1984]. These laborious approaches demonstrated the topographic distribution of bivalents in the spermatocyte nucleus of various species, including human and mouse [von Wettstein et al., 1984]. Currently, most of the reports nicely identify the SCs through immunocytochemistry addressed to main constituent of the CE or LEs (SYCP1 or SYCP3 proteins, respectively). This method may be applied in squashed spermatocytes where nuclei preserve their sphericity, or in spermatocyte spreads where the $3 \mathrm{D}$ organization of the nucleus is lost but bivalents keep their interactions [Berrios et al., 2014].

Thus, the nuclear organization of spermatocytes in meiotic prophase I is primarily determined by the synaptic organization of the bivalents bound by their telomeres to the nuclear envelope and described as arc-shaped trajectories through the $3 \mathrm{D}$ nuclear space. However, from this basic meiotic organization, a nuclear architecture arises that is conditioned by the individual characteristics of the chromosomes and the opportunity for interactions between their domains [Berrios et al., 2014].

In this context the organization of the mouse spermatocyte nucleus at meiotic prophase I is reviewed here.

\section{Chromosome Topology in Mouse Spermatocytes}

Given the characteristics previously described in meiotic prophase spermatocytes, it is possible to identify bivalents in fully transcriptionally active nuclei. Following the path of each SC, it is also feasible to observe the arrangement of bivalents and their sequentially ordered domains in the nuclear space [Fernández-Donoso and Berrios, 1985]. 


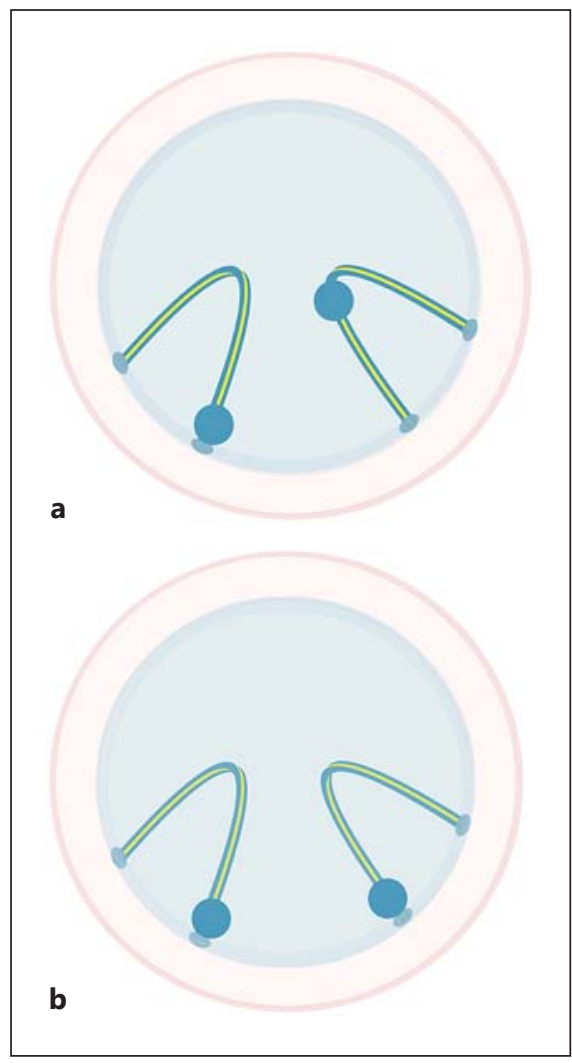

Fig. 1. Meeting probability between 2 chromosomal domains according to the nuclear space they occupy in a spermatocyte at meiotic prophase. Bivalents are only represented by the synaptonemal complex. a Pachytene nucleus with one telocentric and one metacentric bivalent. The centromeric region of a telocentric bivalent will be localized at the nuclear periphery, whereas the centromeric region of a metacentric bivalent will be localized towards the nucleus center. The probability of encounter between these domains is very low. b Pachytene nucleus with 2 telocentric bivalents. The centromeric regions of both telocentric bivalents will be localized at the nuclear periphery. The probability of encounter between these domains is higher.

The length and morphology of chromosomes play an obvious role in meiotic nuclear organization. For instance, if we compare the nuclear location of the centromeric region between 2 bivalents of similar size, one telocentric and the other metacentric, we will find that both bivalents describe arcs of similar size. However, in the first, the centromeric region will be located at the nuclear periphery next to the nuclear envelope, but in the other, the centromeric region will be placed toward the nucleus center (Fig. 1a). The odds of meeting and association between these centromeric regions will be very low. However, the odds could be higher if both chromosomes are telocentric, even if they are of different sizes (Fig. 1b). This finding is not trivial, because a pachytene spermatocyte nucleus that is approximately $14 \mu \mathrm{m}$ of diameter presents a nuclear envelope surface of approximately 620 $\mu \mathrm{m}^{2}$ and a 3-dimensional space of approximately 1,400 $\mu \mathrm{m}^{3}$ where in any of both spaces the likelihood of meeting between 2 centromeric regions each of $1 \mu \mathrm{m}^{3}$ would be very unlikely [Berrios et al., 2010]. Taking these considerations into account, we have proposed that the real origin of chromosome domain associations should occur within the bouquet at early prophase.

\section{The Constitutive Heterochromatin}

Heterochromatin has been classified into facultative and constitutive heterochromatin. Facultative heterochromatin refers to a type of chromatin that may form at various chromosomal regions that generally contain genes that must be kept silent upon developmental cues. In contrast, constitutive heterochromatin has a low density of protein-coding genes and is transcribed at various stages of development to produce transient noncoding RNA of varied functions. In most organisms, heterochromatin is a large part of the genome, and the bulk of constitutive heterochromatin forms at pericentromeric regions and at the telomeres. These gene-poor areas are typically made of tandem repetitions, also named satellites, which vary in size from $5 \mathrm{bp}$ (microsatellites) to a few hundred bp [Hennig, 1999; Eymery et al., 2009; Saksouk et al., 2015]. Heterochromatin is characterized by typical posttranslational modification profiles on histones, the most prominent being global hypoacetylation leading to chromatin fiber compaction. In addition, a typical mark of constitutive heterochromatin is the trimethylation of histone $\mathrm{H} 3$ on lysine 9 (H3K9me3) [Saksouk et al., 2015]. Heterochromatin protein 1 (HP1) is a family of small proteins that recognize the $\mathrm{H} 3 \mathrm{~K}-$ $9 \mathrm{me} 2 / 3$ mark and contribute to the formation and propagation of transcriptionally inactive heterochromatin [Tamaru and Selker, 2001; Dillon, 2004]. Constitutive heterochromatin tends to associate, forming chromocenters that are easily visible by light microscopy as they stain heavily [Schmid et al., 1975; Mayfield and Ellison, 1975]. Heterochromatin plays important roles in global genome organization [Politz et al., 2015] and in meiosis by shaping relatively stable unions between different bivalents [Redi et al., 2001]. In spermatocytes, constitutive heterochromatin forms one or more condensed chromocenters that appear as clusters of chromatin denser than the oth- 

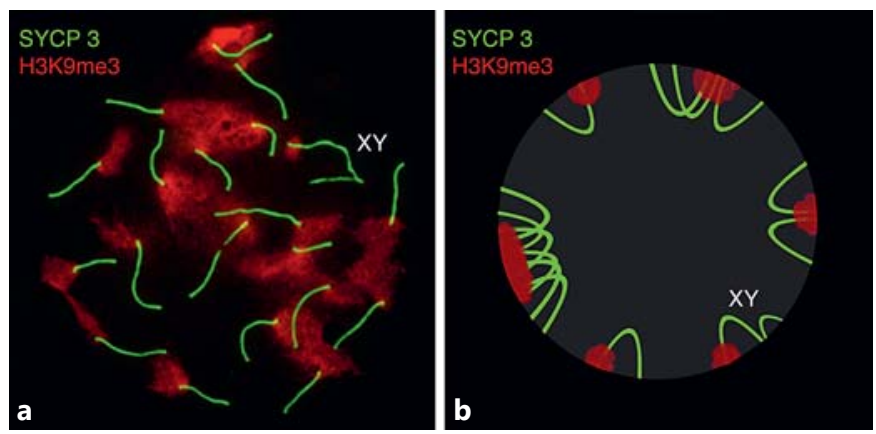

Fig. 2. Heterochromatin domains in single and associated bivalents of a mouse pachytene nucleus. a Nuclear microspread of a pachytene spermatocyte exhibited well-preserved associations mediated by constitutive heterochromatin. Different numbers of single or associated 19 autosomal bivalents are noted. The XY bivalent is indicated. The chromosomal axes were labelled with FITC anti-SYCP3 antibodies (green) and the constitutive heterochromatin with Texas Red anti-H3K9me3 antibodies (red). b Representation of the nucleus corresponding to the microspread of $\mathbf{a}$. Although the bivalents are widely distributed on the inner surface of the nuclear envelope, the larger diameter circumference of the nucleus was only represented in this $2 \mathrm{D}$ drawing. The bivalents appear single or associated through constitutive heterochromatin and are localized at the periphery of the pachytene nucleus. The partially synapsed $\mathrm{X}$ and $\mathrm{Y}$ chromosomes appear attached to the nuclear envelope but without association with any autosomal bivalent.

er nuclear components [Schwarzacher et al., 1984; Berrios et al., 1999, 2004; Dillon, 2004]. Heterochromatin has also played an important role in the evolution and differentiation of $\mathrm{X}$ and $\mathrm{Y}$ sex chromosomes by limiting their pairing and synapsis in meiosis [Graves, 2006].

In Mus musculus domesticus $(2 \mathrm{n}=40)$, all chromosomes are telocentric, with large blocks of pericentromeric heterochromatin surrounding the centromere and extending towards the proximal telomere [Pardue and Gall, 1970; Redi et al., 2001]. In the centromeric regions of Mus, there are 2 types of repetitive DNA sequences: major and minor satellites [Garagna et al., 2002]. The minor satellite is associated with the functioning of the centromere itself, whereas the major satellite composes the heterochromatin responsible for chromosome aggregation in clusters or chromocenters [Joseph et al., 1989; Garagna et al., 2002; Guenatri et al., 2004]. Therefore, in Mus 2n $=40$ spermatocytes, the centromeric and pericentromeric heterochromatin of all bivalents is located throughout the nuclear envelope at the nuclear periphery [Brinkley et al., 1986; Berrios et al., 1999, 2004]. Several chromocenters of different size may be present in each pachytene nucleus according to the number of associated bivalents as it can be seen on a spreading of a pachytene nucleus where bivalent associations mediated by heterochromatin were preserved (Fig. 2). At the leptotene/zygotene transition of the meiotic prophase, telomeres form a polarized organization called the bouquet that is essentially contemporaneous with the onset of pairing, synapsis, and SC formation [Zickler and Kleckner, 1998; 2015; Harper et al., 2004]. During the bouquet configuration of mouse spermatocytes, the physical closeness of the proximal telomeres and pericentromeric heterochromatin may result in the transient formation of a large heterochromatin chromocenter located at the nuclear periphery (Fig. 3a). Thus, the chromocenters that are present later in the pachytene nuclei would be the result of the random disaggregation of the large chromocenter or the only heterochromatin cluster formed during the bouquet (Fig. 2b, 3b). During pachynema, SCs exhibit dramatic and continuous movements throughout the nucleus, traversing relatively large distances while twisting, folding, and unfolding [Scherthan, 2009; Shibuya et al., 2014; Lee et al., 2015]. Chromosomal displacement and movements may contribute to the contact among chromosomal territories; however, these movements in combination with the increased size of the nuclear volume may contribute to the disaggregation of the bivalent interactions produced within the bouquet [Berrios et al., 2010]. Quantitative results have demonstrated that the pericentromeric regions of the 19 autosomal bivalents are associated within each Mus spermatocyte in a roughly similar fashion to the potential combinations of 19 equivalent elements dispersed randomly over a limited area of the nuclear envelope [López-Fenner et al., 2014]. The XY bivalent occupies the peripheral space of the nucleus due to binding to the nuclear envelope of telomeres and condensed chromatin; however, the XY bivalent does not generally establish associations with other chromosomes. In a similar manner as in Mus spermatocytes, the chromocenters in spermatocytes of various species of mammals aggregate in a species-specific pattern according to the chromosomal distribution of pericentromeric heterochromatin [Solari and Tres, 1970; Berrios et al., 1999]. Through serial sections of well-preserved nuclei, a central distribution of chromocenters was observed in pachytene spermatocyte nuclei of Ctenomys opimus ( $2 \mathrm{n}=26$; Rodentia, Ctenomidae) and Octodon degus ( $2 \mathrm{n}=58$; Rodentia, Octodontidae), whereas a peripheral distribution was observed in pachytene spermatocyte nuclei of Pudu puda $(2 \mathrm{n}=70$; Artiodactyla, Cervidae). However, in the spermatocyte nuclei of 2 South American marsupial species, Thylamys elegans ( $2 \mathrm{n}=14$; Didelphidae, Marsupialia) and Dromiciops
64

Cytogenet Genome Res 2017;151:61-71 DOI: $10.1159 / 000460811$
Berrios 
Fig. 3. Nuclear territories of associated chromosomal domains in pachytene nuclei considering chromosome morphology and the bouquet of early meiotic prophase. a Chromosome configuration at the bouquet of a spermatocyte bearing 7 telocentric bivalents. The chromosomes share all the heterochromatic regions in a reduced peripheral nuclear space where they become associated. b The associated bivalents observed later at the pachytene nucleus would be the result of the random disaggregation of the only heterochromatin cluster formed during the bouquet. This case was represented by a pachytene nucleus with one cluster of 4 associated bivalents and the other with 2 and a single bivalent. c Chromosome configuration at the bouquet of a spermatocyte bearing a mixed karyotype with 3 telocentric and 2 metacentric chromosomes. The chromosomes are localized together presenting the heterochromatic regions in 2 different spaces for association: one among heterochromatins of telocentric bivalents at the nuclear periphery and another among heterochromatins of metacentric bivalents toward the nucleus center. $\mathbf{d}$ The associated bivalents observed later at the pachytene nucleus would be the result of the random disaggregation of both: the heterochromatin cluster of the metacentric chromosomes that are mainly located at the nucleus center and the heterochromatin cluster of the telocentric chromosomes located at the nuclear periphery. This case was represented by a pachytene nucleus with 2 associated telocentric bivalents, 1 single telocentric bivalent and 2 associated metacentric Rb.

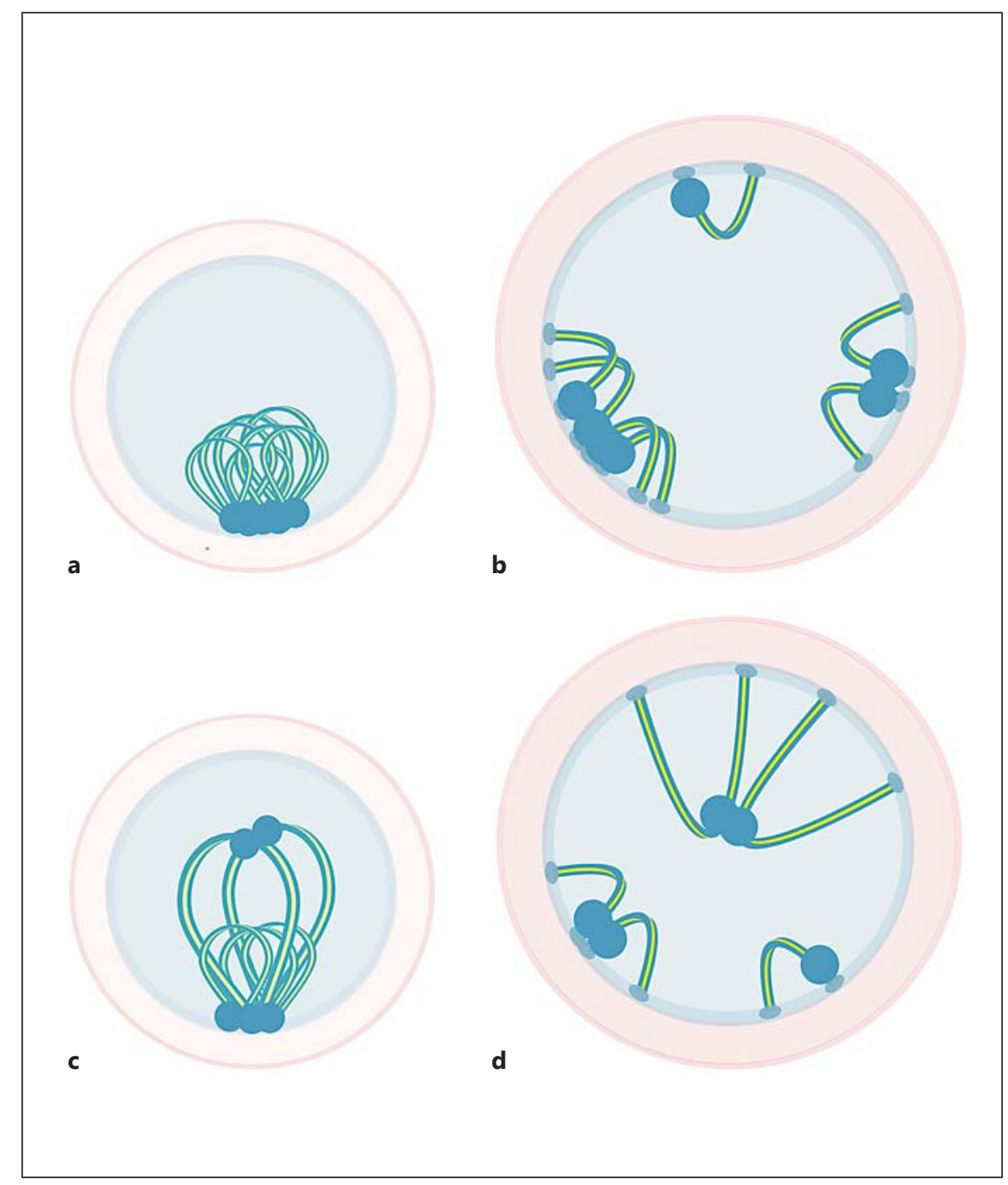

gliroides $(2 \mathrm{n}=14$; Microbiotheriidae, Marsupialia), which exhibited no heterochromatin, chromocenters or associations between bivalents were not observed [Berrios et al., 1999].

\section{The Nucleoli}

The nucleolus is the morphologic picture of active gene expression. It is an essential nuclear organelle that is crucial for cell survival and has a primary role in processing and assembling of ribosomes. Further investigations in somatic cells have revealed that the nucleolus has a more dynamic character and diverse functions [Scheer and Hock, 1999; Raska et al., 2006; Németh and Längst,
2011]. The nucleolus organizer region (NOR) contains tandemly repeated ribosomal DNAs (rDNAs) and is embedded in heterochromatin in most eukaryotes. It is surprising that rDNAs produce the overwhelming majority of RNAs in the cell despite its association with 'silenced' heterochromatin. This paradox suggests that the evolutionarily conserved positioning of NORs in heterochromatin may regulate important, unknown features of nucleolus formation [Peng and Karpen, 2009]. For instance, a mammalian nucleolar remodeling complex (NoRC) regulates heterochromatin formation and rRNA expression by controlling histone $\mathrm{H} 4$ deacetylation, H3K9 dimethylation, and de novo DNA methylation of rDNA [Santoro et al., 2002]. Nucleolar chromosomes contain NORs and consequently may build the nucleolus. As not- 
ed above, in the karyotype of several species, NORs appear to be flanked by pericentromeric heterochromatin. Therefore, in the nuclei of most spermatocytes, nucleoli are linked to the NOR from which they originated and are surrounded by constitutive heterochromatin [Schwarzacher et al., 1984; Berrios et al., 2004]. This is the case of the 5 pairs of human acrocentric nucleolar chromosomes that bear NORs and heterochromatin in their short arms. Consistently, spermatocytes contain 5 nucleolar bivalents, with NORs and heterochromatin localized at the nuclear periphery. These bivalents are often associated with each other in groups of $2,3,4$, or even 5 , forming a common nucleolus settled in heterochromatin [Stahl et al., 1984; Berrios and Fernández-Donoso, 1990]. A similar case is noted with nucleolar chromosomes and nuclear organization of Mus spermatocytes. Mus also has 5 pairs of nucleolar chromosomes, but NORs are localized in the pericentromeric region of the long arms of telocentric chromosomes [Cazaux et al., 2011; Britton-Davidian et al., 2012]. Thus, 5 nucleolar bivalents are also present in spermatocytes of Mus $2 \mathrm{n}=40$, with the NOR and heterochromatin localized at the nuclear periphery. Nucleolar bivalents appear to be associated with other bivalents and among themselves through pericentromeric heterochromatin. Through electron microscopy of thin sections of well-preserved nuclei, a similar nuclear localization was observed in spermatocytes of Phyllotys osgoodi (Rodentia, Muridae), where the terminal NORs of 4 nucleolar bivalents give rise to peripheral nucleoli but are not associated with them [Berrios et al., 2004]. The intercalated NORs of the single nucleolar bivalent from O. degus (Rodentia, Octodontidae), C. opimus (Rodentia, Ctenomidae), and Chinchilla lanigera (Rodentia, Cavidae) give rise to a central nucleolus, but only the first 2 are surrounded by associated heterochromatin from various bivalents [Berrios et al., 2004]. In thin sections of nuclei from fixed spermatocytes of the marsupial species T. elegans (Didelphidae, Marsupialia), the terminal NOR in one subtelocentric pair gives rise to a peripheral nucleolus. In D. gliroides (Microbiotheridae, Marsupialia), the terminal NORs in 2 pairs of subtelocentric chromosomes give rise to 2 peripheral nucleoli [Berrios et al., 2004]. Clearly, the nuclear localization of the nucleoli depends on the position of NORs in nucleolar chromosomes, but the association among nucleolar chromosomes mainly depends on the presence of constitutive heterochromatin. Similar relationships have been observed in nuclear microspreads of Peromyscus spermatocytes where nucleolar bivalents appear with nucleoli but associated among them by heterochromatin [Greenbaum et al., 1986]. After studying numerous animal and plant species, it was found that approximately $90 \%$ of NORs are located on a short arm, and more than $80 \%$ are located near the telomeric region, suggesting that an optimal location of NORs within the arms of nucleolar chromosomes exists [Lima-deFaria, 1999]. If most NORs were located near the telomeric region, it would imply that most of the nucleoli should be located at the periphery of the spermatocyte nucleus. It would be bold to hypothesize about the biological significance of a peripheral or central, a single or multiple, simple or associated nucleolus. What is evident, however, is that nucleoli are part of complex nuclear territories that are nonrandomly distributed in spermatocyte nuclei.

\section{Consequences of Associations of Heterochromatin Blocks}

In mouse telocentric chromosomes, telomere/centromere sequences exhibit the same polarity and a high sequence identity of $>99 \%$ between nonhomologous chromosomes [Garagna et al., 2001]. The homology of satDNA sequences shared by the telocentric mouse chromosomes potentially occurred via multiple small exchanges among these large tracts of tandemly repeated DNA [Kalitsis et al., 2006].

The associations among the autosomal bivalents during meiotic prophase, which occur precisely through their heterochromatic domains, would also provide a scenario in which these frequent recombination exchanges between nonhomologous chromosomes may have occurred and led to concerted evolution [Hamilton et al., 1990; Plohl et al., 2008]. Subsequently, these recombined chromosomes would be passed on to the offspring through the resulting gametes, thus representing a mechanism for the sequence homogenization that would occur over cell generations. The relationship between concerted evolution and chromosome associations has also been proven with the bimodal karyotype of the domestic pig. The diploid set of $2 \mathrm{n}=38$ elements comprises 12 acrocentric and 24 (sub-)metacentric autosomes in addition to the sex chromosomes. A homogeneous satellite DNA family characterizes the acrocentric subset, whereas different satellites are distributed on the bi-armed chromosomes [Jantsch et al., 1990].

Homologous recombination among the densely packed repetitive sequences of the pericentromeric heterochromatin is strongly restricted, as demonstrated in mouse spermatocytes. The SMC5/6 complex, a member
66

Cytogenet Genome Res 2017;151:61-71 DOI: $10.1159 / 000460811$
Berrios 
of the structural maintenance of chromosome (SMC) family, is localized at the pericentromeric heterochromatin, repressing meiotic recombination around the centromeres via a mechanism yet to be elucidated. Therefore, SMC5/6 might be responsible for preventing aberrant recombination events in these high-risk regions that could lead to anomalies and genomic instability [Verver et al. 2013].

The frequent random associations among all autosomal bivalents in Mus spermatocytes would be consistent with the necessary physical proximity among their heterochromatic regions for Robertsonian fusions to occur among any of the 19 bivalents, as described for the different subspecies of Mus [Redi and Capanna, 1988].

\section{Robertsonian Chromosomes}

Robertsonian $(\mathrm{Rb})$ translocations involve DSBs at the centromere level in 2 telocentric (acrocentric) chromosomes followed by repair (fusion), ligating the respective long arms creating a metacentric $\mathrm{Rb}$ chromosome. The short arms of the original telocentric chromosomes, including the proximal telomeres, part of the satellite DNA, and frequently 1 centromere, are lost [Comings and Avelino, 1972; Nanda et al., 1995; Garagna et al., 2001, 2002].

The homogeneity of telocentric chromosomes of $M$. musculus domesticus $2 \mathrm{n}=40$ and the numerous subspecies with reduced diploid numbers and carrying metacentric Rb chromosomes [Piálek et al., 2005] make this species a convenient model to evaluate the influence of chromosome morphology on the nuclear architecture of spermatocytes.

In the bouquet of the $2 n=24$ spermatocytes, which are carriers of $8 \mathrm{Rb}$ bivalents, it is remarkable that 2 different areas for association are characteristic for these nuclei: one among the heterochromatins of telocentric bivalents at the nuclear periphery and another among the heterochromatins of metacentric bivalents toward the center of the nucleus (Fig. 3c, d) [Berrios et al., 2014; Garagna et al., 2014]. Therefore, at pachynema, the pericentromeric regions of metacentrics are mainly located in the nuclear interior, forming only a few chromocenters (1-2 in spermatocytes carrying $2 \mathrm{n}=24$ chromosomes), and the pericentromeric regions of telocentric chromosomes remain close to the nuclear periphery. This new nuclear architecture would favor the progressive fusion of the remaining telocentric chromosomes. It is also possible that this narrowing of association opportunities is related to the over-

Nuclear Architecture of Mouse Spermatocytes all trend toward the production of metacentric chromosomes, as observed in the chromosomal evolution of this species [King, 1993; Searle, 1998]. On the other hand, the association between the heterochromatic regions of metacentric bivalents could be the scenario in which WARTS (whole-arm reciprocal translocation) or the whole chromosomal arms interchange between metacentric $\mathrm{Rb}$ may occur [Capanna and Redi, 1995; Solano et al., 2007]. The described topological nuclear organization allows frequent contact between chromosomal domains that may eventually experience rearrangements. However, it is also necessary to consider that during meiotic prophase, a programmed induction of DSBs leads to the exchange of genetic material between homologous chromosomes [Baudat et al., 2013]. Therefore, under this topographic scenario, the full DNA repair machinery would be available, which could account for heterologous DNA exchange that may modify the structure of the involved chromosomes. Notwithstanding, the actual occurrence of rearrangements depends, inter alia, on the characteristics of chromosomes and mainly on the presence of heterochromatin. In summary, the nuclear architectural features together with the intense DNA nicking and repair activity of first meiotic prophase [Neale and Keeney, 2006] can set the stage for chromosome interactions and create the conditions for eventual chromosomal rearrangements.

Given that the nucleolar chromosomes of Mus $2 \mathrm{n}=40$ bear NORs in the proximal long arm, the derived $\mathrm{Rb}$ metacentric chromosomes do not lose the NORs (as they occur in $\mathrm{Rb}$ translocations between human nucleolar chromosomes). Therefore, it is possible to study the nuclear territories composed of heterochromatin and nucleoli in spermatocytes of Mus $2 \mathrm{n}=24$ homozygous for $\mathrm{Rb}$. The nucleolar chromosomes in Mus $2 \mathrm{n}=40$ consist of 5 telocentric pairs $(12,15,16,18$, and 19), whereas 3 derived metacentrics (Rb: 10;12, 5;15, and 16;17) and 2 telocentrics (18 and 19) are noted in Mus 2n = 24 [Cazaux et al., 2011; Britton-Davidian et al., 2012]. In shaping these territories, nucleolar telocentrics appear to be associated with other telocentrics and nucleolar metacentrics with other metacentric chromosomes through pericentromeric heterochromatin. A preferential association was not observed between nucleolar bivalents but between morphologically similar chromosomes (Fig. 4a, c). Nucleolar expression, as estimated by the fibrillarin area, was very variable between all types of nucleolar bivalents. It does not seem that the expression of NORs located within the nucleolar telocentrics is different from NORs integrated in nucleolar $\mathrm{Rb}$ metacentric bivalents. In both cases, the

Cytogenet Genome Res 2017;151:61-71 DOI: $10.1159 / 000460811$ 


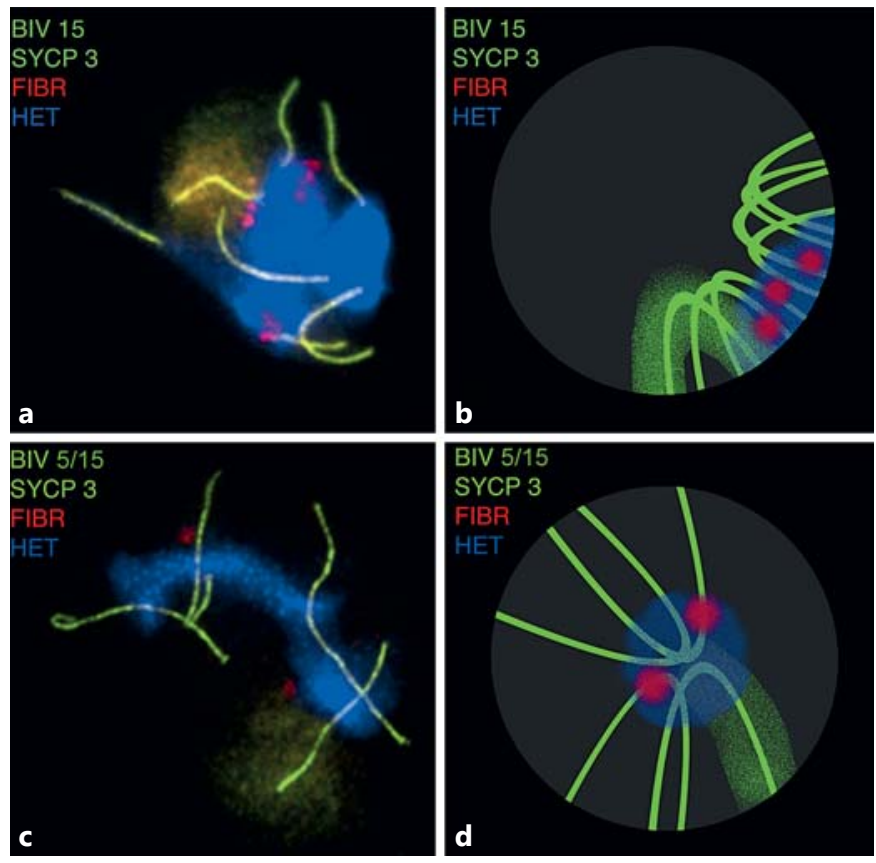

Fig. 4. Nuclear territories formed by heterochromatin and nucleoli in the spermatocyte nucleus of Mus $2 \mathrm{n}=40$ and homozygote $\mathrm{Rb} 2 \mathrm{n}=24$. The chromosomal axes were labeled with FITC antiSYCP3 antibodies (green), nucleoli with Texas Red anti-fibrillarin antibodies (red), and the constitutive heterochromatin was stained with DAPI (blue). a Chromosomal territory composed of 7 telocentric bivalents associated among them by their constitutive heterochromatin. The nucleolus produced by the nucleolar bivalent 15 and the nucleoli from other 2 nucleolar bivalents form part of this nuclear territory. The chromatin of the nucleolar bivalent 15 by FISH painting and specific DNA probe was identified. b Representation of the nucleus corresponding to the microspread of $\mathbf{a}$. The 7 autosomal bivalents associated through constitutive heterochromatin should be localized at the periphery of the pachytene nucleus. Three of them are nucleolar bivalents, and their nucleoli are immersed within the heterochromatin cluster. The nucleolar bivalent 15 is forming part of this nuclear territory. c Chromosomal territory composed by 4 metacentric Rb bivalents associated among them by their constitutive heterochromatin. The nucleolus produced by the nucleolar bivalent $5 / 15$, and the nucleoli from other nucleolar bivalents form part of this nuclear territory. The chromatin of the chromosomal arm 15 of the nucleolar bivalent $5 / 15$ by FISH painting and specific DNA probe was identified. d Representation of the nucleus corresponding to the microspread of c. The 4 metacentric bivalents associated through constitutive heterochromatin should be localized at the center of the pachytene nucleus. Two of them are nucleolar bivalents and their nucleoli are immersed within the heterochromatin cluster. The nucleolar bivalent 5/15 forms part of this nuclear territory. The nucleoli are immersed within the heterochromatin cluster but not directly associated with them. nucleoli occur embedded in territories formed by heterochromatin originating from the union or association between variable numbers of bivalents, which should be located at the periphery or at the center of the nucleus (Fig. 4b, d). In this respect, it is possible that frequent and random associations of heterochromatin, in which the nucleolar bivalents participate, are the most favorable functional organization. However, these chromosomal configurations are also favorable to propagate rDNA toward the heterochromatin of nonnucleolar chromosomes as has been described in various strains of Mus [Rowe et al., 1996; Ito el al., 2008; Britton-Davidian et al., 2012]. Given the condensed nature of the heterochromatin structure and its association with silencing complexes, it is generally believed that heterochromatin silences the expression of genes embedded in it by limiting the access of DNA to transcription factors. However, this notion is challenged by the nucleolar activity in heterochromatin.

\section{Nuclear Architecture: Stability or Change?}

Constitutive heterochromatin is unquestionably a key element in the nuclear architecture of spermatocytes; however, heterochromatin is also proposed as responsible for chromosomal and genomic stability [Politz et al., 2015] and credited to be responsible for chromosome change and the evolution of genomes [Plohl et al., 2008]. Arguably, heterochromatin participates in both situations, and the explanation for these seemingly disparate roles perhaps could be in noncoding RNAs and transposons in heterochromatin DNA sequences [Kazazian, 2004; Rangan et al., 2011]. At this level of analysis, this assumption cannot be tested; however, in this context some evidence from other authors can be discussed. For instance, with the increased sensitivity of molecular techniques, transcription of pericentromeric satellite repeats has been confirmed in a multitude of organisms and in various contexts, including proliferation, development, differentiation, senescence, stress response, and transformation [Saksouk et al., 2015]. On the other hand, there is a subfamily of proteins (Piwi) expressed almost exclusively in gonadal tissues, often only in germline cells, where they form the core of animal transposon silencing pathways [Siomi et al., 2011]. Piwi proteins interact with piRNAs (Piwi-interacting RNAs) that appear to be generated from a small number of long single-stranded RNA precursors often encoded by heterochromatic repetitive DNA sequences. Piwi/piRNAs play crucial roles during germline development, meiosis, spermiogenesis, and
Berrios 
transposon silencing [Siomi et al., 2011; Thomson and Lin, 2009; Joshua-Tor and Hannon, 2011]. These functions may be achieved by diverse epigenetic mechanisms, including histone modifications, de novo DNA methylation of transposons, and posttranscriptional regulation, such as destabilizing retrotransposon RNAs via endonucleolytic cleavage [Crichton et al., 2014]. Research also provides evidence that Piwi proteins and piRNAs are not only crucial for transposon silencing in the germline but also mediate novel mechanisms of epigenetic programming, DNA rearrangements, mRNA turnover, and translational control in the germline [ $\mathrm{Ku}$ and Lin, 2014]. The underlying heterogeneity in constitutive heterochromatin added to its important role in the interaction between chromosomal domains and would confer to a great versatility of functions and consequences for the nuclear architecture of spermatocytes.

\section{Concluding Remarks}

The nuclear architecture of spermatocytes of M. musculus domesticus and other mammalian species is a higher order pattern of spatial associations among chromosomal domains from different bivalents that follows the rules of meiotic prophase organization and is also consistent with the morphology of chromosomes. Both features determine the nuclear localization of chromosomal domains and thus their likely encounters and interactions.

The nuclear architecture proposal does not intend to regulate the order of the nucleus in meiotic prophase I. On the contrary, it takes advantage of the circumstances that characterize this meiotic stage at which we can distinguish chromosomes in a functional nucleus. With various techniques for in situ identification applied to meiotic prophase nuclei observed through a microscope, we can scrutinize chromosomal domains or entire chromosomes as well as their localization and interactions, thus revealing a physical setting that can be compatible with the evidence provided from other areas regarding genomic function. For instance, to better understand gene interactions, regulation of gene expression, variegation, and other phenomena, it can be useful to have a scenario of possible meetings between chromosomal domains or genomic regions involved [Shatskikh and Gvozdev, 2013].

Considering that the nuclear architecture of meiotic prophase would be based on the characteristics of chromosomes and their chances of meeting, it is reasonable to take into account that all nuclei of spermatocytes of the same organism and individuals of the same species will be relatively similar. If we further consider that these are the cells that will give rise to gametes, we can expect that any major or minor genomic change may be repeated and eventually inherited by many descendants. This phenomenon would not be a minor fact as it may help to explain the high relative speed of propagation of some genomic or chromosomal changes in a reproductive community.

In this sense, it would be the nuclear genome itself and its nuclear organization that would favor its own variation over time. Natural selection could only act over the innovations produced by the genome itself.

\section{Acknowledgment}

This work was supported by FONDECYT Project from CONICYT (Chile).

\section{Disclosure Statement}

The author has no conflicts of interest to declare.

\section{References}

Baudat F, Imai Y, de Massy B: Meiotic recombination in mammals: localization and regulation. Nat Rev Genet 14:794-806 (2013).

Berríos S, Fernández-Donoso R: Nuclear architecture of human pachytene spermatocytes: quantitative analysis of associations between nucleolar and XY bivalents. Hum Genet 86: 103-116 (1990).

Berríos S, Ayarza E, Moreno M, Paulos A, Fernández-Donoso R: Non-random distribution of the pericentromeric heterochromatin in mei- otic prophase nuclei of mammalian spermatocytes. Genetica 106:187-195 (1999).

Berríos S, Fernández-Donoso R, Pincheira J, Page J, Manterola M, Cerda MC: Number and nuclear localisation of nucleoli in mammalian spermatocytes. Genetica 121:219-228 (2004).

Berríos S, Manterola M, Prieto Z, López-Fenner J, Page J, Fernández-Donoso R: Model of chromosome associations in Mus domesticus spermatocytes. Biol Res 43:275-285 (2010).
Berríos S, Manieu C, López-Fenner J, Ayarza E, Page J, et al: Robertsonian chromosomes and the nuclear architecture of mouse meiotic prophase spermatocytes. Biol Res 47:16-29 (2014).

Bisig CG, Guiraldelli MF, Kouznetsova A, Scherthan H, Höög C, et al: Synaptonemal complex components persist at centromeres and are required for homologous centromere pairing in mouse spermatocytes. PLoS Genet 8:e1002701 (2012).
Nuclear Architecture of Mouse Spermatocytes
Cytogenet Genome Res 2017;151:61-71 DOI: $10.1159 / 000460811$ 
Boateng KA, Bellani MA, Gregoretti IV, Pratto F, Camerini-Otero RD: Homologous pairing preceding SPO11-mediated double-strand breaks in mice. Dev Cell 24:196-205 (2013).

Bolcun-Filas E, Costa Y, Speed R, Taggart M, Benavente $\mathrm{R}$, et al: SYCP2 is required for synaptonemal complex assembly, DSB repair and homologous recombination. J Cell Biol 176: 741-747 (2007).

Brinkley BR, Brenner SL, Hall JM, Tousson A, Balczon RD, Valdivia MM: Arrangements of kinetochores in mouse cells during meiosis and spermiogenesis. Chromosoma 94:309317 (1986).

Britton-Davidian J, Cazaux B, Catalan J: Chromosomal dynamics of nucleolar organizer regions (NORs) in the house mouse: microevolutionary insights. Heredity 108:68-74 (2012).

Burgoyne PS: Genetic homology and crossing over in the $\mathrm{X}$ and $\mathrm{Y}$ chromosomes of mammals. Hum Genet 61:85-90 (1982).

Burgoyne PS, Mahadevaiah SK, Turner JM: The consequences of asynapsis for mammalian meiosis. Nat Rev Genet 10:207-216 (2009).

Capanna E, Redi CA: Whole-arm reciprocal translocation (WART) between Robertsonian chromosomes: finding of a Robertsonian heterozygous mouse with karyotype derived through WARTs. Chromosome Res 3:135137 (1995).

Cazaux B, Catalan J, Veyrunes F, Douzery E, Britton-Davidian J: Are ribosomal DNA clusters rearrangement hotspots? A case study in the genus Mus (Rodentia, Muridae). BMC Evol Biol 11:124-138 (2011).

Comings DE, Avelino E: DNA loss during Robertsonian fusion in studies of the tobacco mouse. Nat New Biol 237:199 (1972).

Crichton JH, Dunican DS, Maclennan M, Meehan RR, Adams IR: Defending the genome from the enemy within: mechanisms of retrotransposon suppression in the mouse germline. Cell Mol Life Sci 71:1581-1605 (2014).

Davies OR, Maman JD, Pellegrini L: Structural analysis of the human SYCE2-TEX12 complex provides molecular insights into synaptonemal complex assembly. Open Biol 2: 120099 (2012).

Dillon N: Heterochromatin structure and function. Biol Cell 96:631-637 (2004).

Ding X, Xu R, Yu J, Xu T, Zhuang Y, Han M: SUN1 Is required for telomere attachment to nuclear envelope and gametogenesis in mice. Dev Cell 12:863-872 (2007).

Eymery A, Callanan M, Vourc'h C: The secret message of heterochromatin: new insights into the mechanisms and function of centromeric and pericentric repeat sequence transcription. Int J Dev Biol 53:259-268 (2009).

Fernández-Donoso R, Berríos S: La arquitectura nuclear y su injerencia en la variabilidad del cariotipo, in Fernandez-Donoso R (ed): El Núcleo, Los Cromosomas y La Evolución, pp. 63-122 (Monografía UNESCO, 1985).
Fraune J, Schramm S, Alsheimer M, Benavente R: The mammalian synaptonemal complex: protein components, assembly and role in meiotic recombination. Exp Cell Res 318: 1340-1346 (2012).

Garagna S, Marziliano N, Zuccotti M, Searle JB, Capanna E, Redi CA: Pericentromeric organization at the fusion point of mouse Robertsonian translocation chromosomes. Proc Natl Acad Sci USA 98:171-175 (2001).

Garagna S, Zuccotti M, Capanna E, Redi CA: High-resolution organization of mouse telomeric and pericentromeric DNA. Cytogenet Genome Res 96:125-129 (2002).

Garagna S, Page J, Fernandez-Donoso R, Zuccotti M, Searle JB: The Robertsonian phenomenon in the house mouse: mutation, meiosis and speciation. Chromosoma 123:529-544 (2014).

Geremia R, Boitani C, Conti M, Monesi V: RNA synthesis in spermatocytes and spermatids and preservation of meiotic RNA during spermiogenesis in the mouse. Cell Differ 5: 343-355 (1977).

Graves JA: Sex chromosome specialization and degeneration in mammals. Cell 124:901-914 (2006).

Greenbaum IF, Hale DW, Fuxa KP: The mechanism of autosomal synapsis and the substaging of zygonema and pachynema from deer mouse spermatocytes. Chromosoma 93:203212 (1986).

Guenatri M, Bailly D, Maison C, Almouzni G: Mouse centric and pericentric satellite repeats form distinct functional heterochromatin. J Cell Biol 166:493-505 (2004).

Hale DW, Hedin MC, Smith SA, Sudman PD, Greenbaum IF: The effect of heterochromatin on synapsis of the sex chromosomes of Peromyscus (Rodentia, Cricetidae). Cytogenet Cell Genet 56:48-56 (1991).

Hamer G, Wang H, Bolcun-Filas E, Cooke HJ, Benavente R, Höög C: Progression of meiotic recombination requires structural maturation of the central element of the synaptonemal complex. J Cell Sci 121:2445-2451 (2008).

Hamilton MJ, Honeycutt RL, Baker RJ: Intragenomic movement, sequence amplification and concerted evolution in satellite DNA in harvest mice, Reithrodontomys: evidence from in situ hybridization. Chromosoma 99: 321-329 (1990).

Harper L, Golubovskaya I, Cande WZ: A bouquet of chromosomes. J Cell Sci 117:4025-4032 (2004).

Heyting C: Synaptonemal complexes: structure and function. Curr Opin Cell Biol 8:389-396 (1996).

Hennig W: Heterochromatin. Chromosoma 108: 1-9 (1999).

Hiraoka Y, Dernburg AF: The SUN rises on meiotic chromosome dynamics. Dev Cell 17:598605 (2009).

Ishiguro K, Kim J, Shibuya H, HernándezHernández A, Suzuki A, et al: Meiosis-specific cohesin mediates homolog recognition in mouse spermatocytes. Genes Dev 28:594-607 (2014).
Ito T, Tsuchiya K, Osawa S, Shibata H, Kanda N: Mapping of rRNA gene loci in the mice, Mus musculus molossinus (Japan) and Mus musculus musculus (Russia) by double color FISH. J Vet Med Sci 70: 997-1000 (2008).

Jantsch M, Hamilton B, Mayr B, Schweizer D: Meiotic chromosomes behaviour reflects levels of sequence divergence in Sus scrofa domestica satellite DNA. Chromosoma 99:330335 (1990).

Joseph A, Mitchell AR, Miller OJ: The organization of the mouse satellite DNA at centromeres. Exp Cell Res 183:494-500 (1989).

Joshua-Tor L, Hannon GJ: Ancestral roles of small RNAs: an Ago-centric perspective. Cold Spring Harb Perspect Biol 3:a003772 (2011).

Kalitsis P, Griffiths B, Choo KH: Mouse telocentric sequences reveal a high rate of homogenization and possible role in Robertsonian translocation. Proc Natl Acad Sci USA 103: 8786-8791 (2006).

Kazazian HH Jr: Mobile elements: drivers of genome evolution. Science 303:1626-1632 (2004).

King M: Species Evolution: The Role of Chromosome Change (Cambridge University Press, Cambridge 1993).

$\mathrm{Ku} \mathrm{HY}$, Lin H: PIWI proteins and their interactors in piRNA biogenesis, germline development and gene expression. Natl Sci Rev 1: 205-218 (2014).

Lammers JH, Offenberg HH, van Aalderen M, Vink AC, Dietrich AJ, Heyting C: The gene encoding a major component of the lateral elements of synaptonemal complexes of the rat is related to X-linked lymphocyte-regulated genes. Mol Cell Biol 14:1137-1146 (1994).

Lee CY, Horn HF, Stewart CL, Burke B, BolcunFilas E, et al: Mechanism and regulation of rapid telomere prophase movements in mouse meiotic chromosomes. Cell Rep 11: 551-563 (2015).

Lima-de-Faria A: The chromosome field theory confirmed by DNA and hybridization. Riv Biol 92:513-515 (1999).

López-Fenner J, Berríos S, Manieu C, Page J, Fernández-Donoso R: Bivalent associations in Mus domesticus $2 \mathrm{n}=40$ spermatocytes. Are they random? Bull Math Biol 76:1941-1952 (2014).

Mahadevaiah SK, Costa Y, Turner JM: Using RNA FISH to study gene expression during mammalian meiosis. Methods Mol Biol 558: 433-444 (2009).

Mayfield JE, Ellison JR: The organization of interphase chromatin in Drosophilidae. Chromosoma 52:37-48 (1975).

Meuwissen RL, Offenberg HH, Dietrich AJ, Riesewijk A, van Iersel M, Heyting C: A coiled-coil related protein specific for synapsed regions of meiotic prophase chromosomes. EMBO J 11:5091-5100 (1992).

Nanda I, Schneider-Rasp S, Winking H, Schmid M: Loss of telomeric sites in the chromosomes of Mus musculus domesticus (Rodentia: $\mathrm{Mu}-$ ridae) during Robertsonian rearrangements. Chromosome Res 3:399-409 (1995). 
Neale MJ, Keeney S: Clarifying the mechanics of DNA strand exchange in meiotic recombination. Nature 442:153-158 (2006).

Németh A, Längst G: Genome organization in and around the nucleolus. Trends Genet 27: 149-156 (2011).

Offenberg HH, Schalk JA, Meuwissen RL, van Aalderen M, Kester HA, et al: SCP2: a major protein component of the axial elements of synaptonemal complexes of the rat. Nucleic Acids Res 26:2572-2579 (1998).

Page SL, Hawley RS: The genetics and molecular biology of the synaptonemal complex. Annu Rev Cell Dev Biol 20:525-558 (2004).

Pardue ML, Gall JG: Chromosomal localization of mouse satellite DNA. Science 170:1356-1358 (1970).

Peng JC, Karpen GH: Heterochromatic genome stability requires regulators of histone $\mathrm{H} 3 \mathrm{~K} 9$ methylation. PLoS Genet 5:e1000435 (2009).

Piálek J, Hauffe HS, Searle JB: Chromosomal variation in the house mouse. Biol J Linn Soc 84: 535-563 (2005).

Plohl M, Luchetti A, Meštrović N, Mantovani B: Satellite DNAs between selfishness and functionality: structure, genomics and evolution of tandem repeats in centromeric (hetero) chromatin. Gene 409:72-82 (2008).

Politz JC, Scalzo D, Groudine M: The redundancy of the mammalian heterochromatic compartment. Curr Opin Genet Dev 37:1-8 (2015).

Qiao H, Chen JK, Reynolds A, Hoog C, Paddy M, Hunter N: Interplay between synaptonemal complex, homologous recombination, and centromeres during mammalian meiosis. PLoS Genet 8:e1002790 (2012).

Rangan P, Malone CD, Navarro C, Newbold SP, Hayes PS, et al: piRNA production requires heterochromatin formation in Drosophila. Curr Biol 21:1373-1379 (2011).

Raska I, Shaw PJ, Cmarko D: New insights into nucleolar architecture and activity. Int Rev Cytol 255:177-235 (2006).

Redi C, Capanna E: Robertsonian heterozygotes in the house mouse and the fate of their germ cells, in Daniel A (ed): The Cytogenetics of Mammalian Rearrangements, pp. 315-359 (Alan R. Liss Inc, New York 1988).

Redi CA, Garagna S, Zacharias H, Zuccotti M, Capanna E: The other chromatin. Chromosoma 110:136-147 (2001).
Rowe LB, Janaswami PM, Barter ME, Birkenmeier EH: Genetic mapping of $18 \mathrm{~S}$ ribosomal RNA-related loci to mouse chromosomes 5 , 6, 9, 12, 17, 18, 19, and X. Mamm Genome 7: 886-889 (1996).

Santoro R, Li J, Grummt I: The nucleolar remodeling complex NoRC mediates heterochromatin formation and silencing of ribosomal gene transcription. Nature Genet 32:393-396 (2002).

Saksouk N, Simboeck E, Déjardin J: Constitutive heterochromatin formation and transcription in mammals. Epigenetics Chromatin 8: 3-20 (2015).

Scheer U, Hock R: Structure and function of the nucleolus. Curr Opin Cell Biol 11:385-390 (1999).

Scherthan H: A bouquet makes ends meet. Nat Rev Mol Cell Biol 2:621-627 (2001).

Scherthan H: Analysis of telomere dynamics in mouse spermatogenesis. Methods Mol Biol 558:383-399 (2009).

Schmid M, Vogel W, Krone W: Attraction between centric heterochromatin of human chromosomes. Cytogen Cell Genet 15:66-80 (1975).

Schwarzacher T, Mayr B, Schweizer D: Heterochromatin and nucleolus-organizer-region behaviour at male pachytene of Sus scrofa domestica. Chromosoma 91:12-19 (1984).

Searle JB: Speciation, chromosomes, and genomes. Genome Res 8:1-3 (1998).

Shatskikh AS, Gvozdev VA: Heterochromatin formation and transcription in relation to trans-inactivation of genes and their spatial organization in the nucleus. Biochemistry (Moscow) 78:603-612 (2013).

Shibuya H, Watanabe Y: The meiosis-specific modification of mammalian telomeres. Cell Cycle 13:2024-2028 (2014).

Shibuya H, Morimoto A, Watanabe Y: The dissection of meiotic chromosome movement in mice using an in vivo electroporation technique. PLoS Genet 10:e1004821 (2014).

Siomi MC, Sato K, Pezic D, Aravin AA: PIWIinteracting small RNAs: the vanguard of genome defence. Nat Rev Mol Cell Biol 12:246258 (2011).
Solano E, Castiglia R, Corti M: A new chromosomal race of the house mouse, Mus musculus domesticus, in the Vulcano Island-Aeolian Archipelago, Italy. Hereditas 144:75-77 (2007).

Solari AJ: The behavior of the XY pair in mammals. Int Rev Cytol 38:273-317 (1974).

Solari AJ, Tres LL: The three-dimensional reconstruction of the XY chromosomal pair in human spermatocytes. J Cell Biol 45: 43-53 (1970).

Stahl A, Hartung M, Devictor M, Berge-Lefranc JL: The association of the nucleolus and the short arm of acrocentric chromosomes with the XY pair in human spermatocytes: its possible role in facilitating sex-chromosome acrocentric translocations. Hum Genet 68:173180 (1984).

Tamaru H, Selker EU: A histone H3 methyltransferase controls DNA methylation in Neurospora crassa. Nature 414:277-283 (2001).

Thomson T, Lin H: The biogenesis and function of PIWI proteins and piRNAs: progress and prospect. Annu Rev Cell Dev Biol 25:355-376 (2009).

Turner JM: Meiotic sex chromosome inactivation. Development 134:1823-1831 (2007).

Verver DE, van Pelt AM, Repping S, Hamer G: Role for rodent $S m c 6$ in pericentromeric heterochromatin domains during spermatogonial differentiation and meiosis. Cell Death Dis 4:e749 (2013).

von Wettstein D, Rasmussen SW, Holm PB: The synaptonemal complex in genetic segregation. Annu Rev Genet 18:331-413 (1984).

Wettstein R, Sotelo JR: Electron microscope serial reconstruction of the spermatocyte I nuclei at pachytene. Microscope 6:557-576 (1967).

Yang F, Wang PJ: The Mammalian synaptonemal complex: a scaffold and beyond. Genome Dyn 5:69-80 (2009).

Zickler D, Kleckner N: The leptotene-zygotene transition of meiosis. Annu Rev Genet 32: 619-697 (1998).

Zickler D, Kleckner N: Recombination, pairing, and synapsis of homologs during meiosis. Cold Spring Harb Perspect Biol 18:7 (2015).

Zickler D, Kleckner N: A few of our favorite things: pairing, the bouquet, crossover interference and evolution of meiosis. Semin Cell Dev Biol 54:135-148 (2016).
Nuclear Architecture of Mouse

Spermatocytes
Cytogenet Genome Res 2017;151:61-71

DOI: $10.1159 / 000460811$ 